Wissenschaffliche Taschenbücher

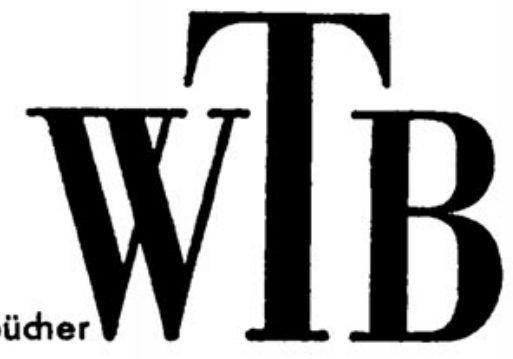

Biologie

Regine Witkowski · Falko H. Herrmann Einführung in die klinische Genetik 


\section{Wissenschaftliche Taschenbücher}

\author{
Eine Auswahl \\ lieferbarer Bände: \\ JOACHM A UTH/DIETMAR GENZOW \\ KLAUS H. HERRMANN \\ Photoelektrische Erscheinungen \\ HANS BANDKMER \\ ANDREAS BELLMANN \\ Wolfhart JUNG/KLAUS RICHTRR \\ Optimale Versuchsplanung
}

FRANK BEICHELT

Prophylaktische Erneuerung

von Systemen

JoACHIM BeLlaCh/PETER FraNKeN

ELKE WARMUTH/WALTER WARMUTH

$M a ß$, Integral

und bedingter Erwartungswert

\section{WOLYRAM BRACER}

HANS-W ALDEMAR STREITWOLF

Theoretische Grundlagen

der Halbleiterphysik

SIEGFRIED BREHMER

Einführung in die MaBtheorie

SIROYRIED BREHMER

Hilbert-Räume und Spektralmaße

JoHn CunxinahaM

Vektoren

GeORG DAtrcourt

Relativistische Astrophysik

K. Ch. Delogarov

Relativitătstheorie und Materialismus

WERNER DUCK

Diskrete Optimierung

\author{
HAXNELORE FISCHER \\ JOACHIM PIEHLER \\ Modellsysteme \\ der Operationsforschung \\ GOTTYKIED FRITZSCHE \\ Grundlagen und Entwurf \\ passiver Analogzwelpole \\ Netzwerke.I \\ Entwurf passiver Analogvierpole \\ Netzwerke II \\ HERHERT GOERING \\ Asymptotische Methoden zur Lösung \\ von Differentialgleichungen \\ HERBERT GORRING \\ Elementare Methoden zur Lösung \\ von Differentialgleichungsproblemen \\ Eddard Herlt/Nikola US SALIÉ \\ Spezielle Relativitätstheorle \\ HeLmut Hess \\ Der elektrische Durchschlag in Gasen
}

V. I. KARPMAN

Nichtlineare Wellen

in dispersiven Medlen

ULRICH KATSMANN

KLAUS Lommatzsch

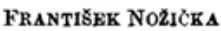

Lineare parametrische Optimierung

A. R. KKSSEL

Akustische Kernresonanz

KONRAD KREHER

Festkörperphysik

DIETER KRESS

Theoretische Grundlagen der Signalund Informationsübertragung 
IHETER LECSCHNER

Grundbegriffe der Thermodynamlk

V. S. LETOCHOW

Laserspektroskopie

DIERCK-EKK KHARD LIEBSCHER

Relativitätstheorie

mit Zirkel und Lineal

WOLFGANG MFILING

Digitalrechner

in der elektronischen Meßtechnik

Teil 1: MeBmethodik .

Teil 2: Gerätetechnik

und Anwendungen

L. I. MIROSCHNITSCHYNKO

Kosmlsche Strahlung

im interplanetaren Raum

P'ETER PALfLek

(itstav E. R. SChL LZF

Physikalische Grundlagen

mechanischer Festkörpereigenschaften

Teil I und II

ULRI'H RÖSEBERf:

Quantenmechanik und Philosoplije

ALBRECHT ROST

Messung

dlelektrischer Stoffeigenschaften

J. V. SACKOV

Wahrscheinlichkeit und Struktur

1.. M. S.AW17K1

Perspektiven der Metallforschung

WOL.FGANG: SCHÄFER

Theoretische Grundlagen

der Stabilität technischer Systeme

ERNST SCHMITZER

Symmetrien und Erhaltungssătze der Physik

Volkmar SCht'richt

Kernexplosionen fur frledliche $\mathbf{Z}$ wecke
NoRbret SIEBER

HANS-PETER LEIDHOLD

Einfuhrung in die Datenverarbeitung

(GerhaRD Wixs('h

Zellulare Systeme

Festkörperphyslk

Entwicklungstendenzen und

Anwendungsmöglichkeiten

Die Schöpfer der physikalischen Optlk

Eine Artikelsammlung

HANS-4; EORo SchöpF

Von Kirchhoff bls Planck

RENATE WAHSNEK

Mensch und Kosmos

Die copernicanische Wende

HELMTT FRIEMEL/JOSEY BROCK

Grundlagen der Immunologle

EBERHARD HOFMAXX

runktionelle Biochemie des Menschen

Band 1 und 2

LOTHAR J̈̈GER

Grundlagen

der Klinischen Immunologle

KARLHEINZ LOHS

DIETKR M ARTINETZ

Entgiftung - Mittel, Methoden

und Probleme.

Joachis Nits'ilMaxs

Entwicklung bei Mensch und Tier

STEPHAN SCHNITZLER

Pharmakologische Aspekte

von Immunreaktlonen

DIETER SPAAR

HELMIT KLEINHEMPEL

HANS JOACHIM MELLER

KLats NacMaNs

Bakteriosen der Kulturpflanzen 


\author{
BHERHARD TKCSCHER \\ Pharmakognosie \\ Teil I-III \\ HRINRI'H BRKMER \\ KLAUS-PETER W ENDI, ANITT \\ Heterogene Katalyse \\ PETKR BIRNNR \\ HANS-JöRg HOFMANN \\ CORNELIT'S WRISS \\ Mo-theoretksche Methoden \\ In der organischen Chemie \\ (ATNTER EPPERT \\ Einfiuhrung \\ In die Schnelle Flüssigchromatographle \\ (ikRHARD (ikISELER/HEINZ SEIDFI. \\ Die Wasserstoffbrückenbindung
}

FalKo H. HERRMANS

MakTINa Ch. HERKMas:

Das Hämoglobin des Menschen

HELYLT HRAPIA

Einführung in die Chromatographie

HANS LCPIA

Grundlagen der Histochemle

Teil l un! II

DIETER ONKE:

Antiblotika -- Chemle und Anwendung

BtRKART P'IHLIPP

(ikRHAR1) RKINISCH

Grundlagen

der makromolekularen Chemie

HORST REMINE RAISFR HERZSCHIH

Massenspektrometrie

in der organischen Chemie

AnOLF \%schiske

Kernmagnetische

Resonanzspektroskople

In der organischen Chemle
Vorschau

‘uf die nächsten Bände:

HORST-HEINO VON BORZESZKOWBEI

RENATE WAHSNER

Newton und Voltaire

Zur Begrondung und,

Interpretation der klassischen

Mechanik

I. A. C'Ol.Ee

Angewandte medizinische

Mikroblologle

WERNER DÖPKE

Dynamische Aspekte der Stereochemle organischer Verbindungen

(:OTTFRIED FRITZS('HE

Entwurf aktiver Analogsysteme

Vetzwerke III

(iERHARI) JAC'KISI'U

Johann Helnrich Lamberts

.Cosmologische Briefe" mit Belträgen zur Frühgeschichte der Kosmologle

WOLFTias: A. KNORRE.

Pharmakokinetik

Theoretische Grundlagen und

praktische Anwendungen

Hasso M BISERT

Fluorchemle

VolkER NOL.LAT

Semi-Markovsche Prozesse

IRAINEK Nixz

Chronopsychophysiologie.

Chronobiologie und Chronomedizin 


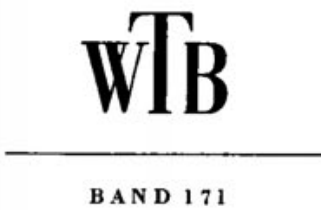

Regine Witkowski

Falko H. Herrmann

\section{Einführung in die klinische Genetik}

2., unveränderte Auflage

Mit 35 Abbildungen und 6 Tabellen 


\section{Reihe B I OLOG I E}

\section{Herausgeber :}

Prof. Dr. H. Bochow, Berlin

Prof. Dr. H. Böhme, Gatersleben

Prof. Dr. H. Borriss, Greifswald

Prof. Dr. E. Hofmann, Leipzig

Prof. Dr. J. O. Hüsing, Rerik

Prof. Dr. U. Taubencek, Jena

Verantwortlicher Herausgeber dieses Bandes:

Prof. Dr. E. Hofmann

Verfasser :

Dr. habil. Regine Witkowski

Dr. sc. nat. Falko H. Herrmann

Berlin/Erfurt

Erschienen im Akademie-Verlag, DDR-108 Berlin.

Leipziger Straße 3-- 4

Lektor: Christiane Grunow

(c) Akademie-Verlag Berlin 19:6

Lizenznummer : $202 \cdot 100 / 558 / 79$

Gesamtherstellung: VEB Druckerei „Thomas Müntzer", 582 Bad Langensalza

Bestellnummer : $7620792(\div 1 / 1) \cdot$ LSV 1324

Printed in GDR

DDR 12,50 M 